\title{
Understanding “Dark” Design Roles in Computing Education
}

\author{
Colin M. Gray \\ gray42@purdue.edu \\ Purdue University \\ West Lafayette, Indiana, USA \\ Kassandra Melkey \\ kmelkey@purdue.edu \\ Purdue University \\ West Lafayette, Indiana, USA
}

\author{
Shruthi Sai Chivukula \\ cshruthi@purdue.edu \\ Purdue University \\ West Lafayette, Indiana, USA \\ Rhea Manocha \\ rmanocha@purdue.edu \\ Purdue University \\ West Lafayette, Indiana, USA
}

\begin{abstract}
In conjunction with the increasing ubiquity of technology, computing educators have identified the need for pedagogical engagement with ethical awareness and moral reasoning. Typical approaches to incorporating ethics in computing curricula have focused primarily on abstract methods, principles, or paradigms of ethical reasoning, with relatively little focus on examining and developing students' pragmatic awareness of ethics as grounded in their everyday work practices. In this paper, we identify and describe computing students' negotiation of values as they engage in authentic design problems through a lab protocol study. We collected data from four groups of three students each, with each group including participants from either undergraduate User Experience Design students, Industrial Engineering students, or a mix of both. We used a thematic analysis approach to identify the roles that students took on to address the design prompt. Through our analysis, we found that the students took on a variety of "dark" roles that resulted in manipulation of the user and prioritization of stakeholder needs over user needs, with a focus either on building solutions or building rationale for design decisions. We found these roles to actively propagate through design discourses, impacting other designers in ways that frequently reinforced unethical decision making. Even when students were aware of ethical concerns based on their educational training, this awareness did not consistently result in ethicallysound decisions. These findings indicate the need for additional ethical supports to inform everyday computing practice, including means of actively identifying and balancing negative societal impacts of design decisions. The roles we have identified may productively support the development of pragmatically-focused ethical training in computing education, while adding more precision to future analysis of computing student discourses and outputs.
\end{abstract}

\section{CCS CONCEPTS}

- Social and professional topics $\rightarrow$ Codes of ethics; Computing education.

\section{(c) (†) (2)}

This work is licensed under a Creative Commons

Attribution-NonCommercial-ShareAlike International 4.0 License.

ICER 2021, August 16-19, 2021, Virtual Event, USA

(C) 2021 Copyright held by the owner/author(s).

ACM ISBN 978-1-4503-8326-4/21/08

https://doi.org/10.1145/3446871.3469754

\section{KEYWORDS}

Ethics, values, dark patterns, ethics education, lab protocol

ACM Reference Format:

Colin M. Gray, Shruthi Sai Chivukula, Kassandra Melkey, and Rhea Manocha. 2021. Understanding "Dark" Design Roles in Computing Education. In Proceedings of the 17th ACM Conference on International Computing Education Research (ICER 2021), August 16-19, 2021, Virtual Event, USA. ACM, New York, NY, USA, 14 pages. https://doi.org/10.1145/3446871.3469754

\section{INTRODUCTION}

Emerging technologies present unique ethical challenges which professionals will need to be properly equipped to engage with to ensure ethically-focused decision making in their fields. While ethics has been discussed in computing, engineering, and technology scholarship for decades through numerous lenses [14, 24, 41], many investigations have previously focused on case studies or other highly-structured pedagogical vehicles (e.g., [9, 13, 19, 45]). However, there is relatively little scholarship that addresses students' pragmatic awareness of ethics as grounded in their work practices.

Numerous HCI and design scholars have identified a variety of means of incorporating ethics in computing education [12, 21, $23,50,54,55]$. Methods and approaches have been designed to teach certain values through fun activities such as designing for privacy [53,60], acknowledging data security [51], highlighting gender representations [3], and incorporating empathy [35], among others. However, Fiesler [24] has also stated that much of tech ethics curricula has been "focused far more on conceptual skills than on specific knowledge," with many educational experiences focused on imparting knowledge about traditional paradigms of ethics or values rather than engaging in the pragmatic tensions that emerge in everyday computing work. These past educational supports frame and support our current work, where we seek to better understand how computing students engage in decisionmaking when confronted with an ethically-nuanced design task.

In this paper, we report on a lab protocol study with 12 undergraduate User Experience Design and Industrial Engineering students, describing how they worked to address an ethically-nuanced task. We used interaction and thematic analysis approaches to analyze the interactions among the participants as they engaged with the task, generating solutions and discussing the rationale behind their decisions. We identified that participants took on a range of manipulative roles which we have categorized as: 1) Solution-focused 
roles: Puppeteer, Nagger, and Diluter; and 2) Rationale-focused roles: fustifier and Capitalist. These pragmatic roles taken on by the participants allow further insight into computing students' ethical awareness, sense of responsibility, and potential future directions that could enhance pragmatic engagement in ethics in the context of computing education.

The contribution of this paper is two-fold: 1) We provide descriptive accounts of the pragmatic roles taken on by computing students as they engage in ethically-nuanced design spaces to inform their ethical awareness, responsibility, and action, building a more detailed perspective on how students engage with ethically-complex decisions; 2) We draw connections between these pragmatic roles and critical concepts such as dark patterns to present implementable directions for pragmatically-focused computing ethics education.

\section{BACKGROUND WORK}

In conjunction with the increasingly ubiquitous role of technology, there is an increase in interest in ethics education to evaluate, imagine, improve, and impact the societal impacts of technology. In the following sections, we will briefly identify some key themes in the accreditation, computing education, and design literature that frame our investigation of computing students' ethical awareness and action.

\subsection{Ethics and Accreditation}

In 1996, the Accreditation Board for Engineering and Technology (ABET) made the integration of professional ethics and into computing curricula mandatory for all accredited computing and engineering programs, beginning in the year 2000. Currently, ABET requires "an understanding of professional, ethical, legal, security and social issues and responsibilities" [1] as part of their outcomebased criteria. However, there is little specific guidance on what form this professional ethics training must take across the curriculum, with some programs focusing on standalone ethics courses and others integrating training across cornerstone, capstone, and other project-based coursework. From a professional organizational perspective, the Association for Computing Machinery (ACM) code of ethics has also incorporated language relevant to computing education curricula in a range of programs $[12,59]$, with a trajectory towards socially responsible computing practices. While there have been debates about the power or role of such codes, studies have shown that the updated ACM code has had little impact on producing actual ethically-centered practices. In a study conducted by McNamara et al. [45], a behavioral ethics study was replicated measuring students' responses to "ethical vignettes"; students in the treatment group were asked explicitly to consider the ACM code as they made decisions, but there was effect on actual decision outcomes as compared with a control group. While there is definite signalling value in the use of codes of ethics (e.g., [6]), there are important limitations in curtailing negative design behaviors that must be addressed through other means of education and support.

\subsection{Ethics Education in Computing Disciplines}

Scholars in the pedagogy of ethics have engaged in a long and ongoing conversation about integrating ethics in computing fields and domains [46] such as HCI [42, 58], Machine Learning [50], Artificial Intelligence Programming [31], and Cybersecurity [9], among others. Recent research has focused attention on the content and role of technology ethics courses, as societal interest in ethics and values has increased, and computing educators are increasingly arguing for ethics to have a more central role in computing curricula (e.g., [30, 32, 56]). As one example, Fiesler, Garrett, and Beard [24] analyzed 115 syllabus from technology ethics courses offered at the university level across domains of design, cybersecurity, privacy, and surveillance. Fiesler and colleagues identified a range of goals for including these courses in required curricula, including: "to teach students to recognize ethical issues in the world," improving communication an argumentative skills, and practically applying "rules" into solution and critiquing to build "good technologies" or "reduce bad things." As another example in a related domain, Lorents and colleagues [43] surveyed undergraduate business majors to understand their ethical attitudes based on presented design scenarios. The results from this study stated that "actions undertaken for profit or malicious intent are judged to be less ethical."

Other pedagogical strategies that have been proposed include the privileging of integrated courses over standalone ethics courses [23, 50], making codes of ethics accessible through quiz shows [12], bringing social issues into classroom activities [54], and incorporating active learning methods such as in-class activities and team projects [54, 55], assignments-focused scaffolding [21], workbooks [60], case study-based curriculum [9], and role-playing activities [53]. Researchers have also suggested various improvements to pedagogical practices, with a focus on how resources to teach ethics could be more grounded in real-world scenarios [24, 39], moving students' knowledge of ethics from conceptual and abstract to pragmatically useful in their everyday work.

\subsection{Tools to Support Ethical Decision-Making}

Increasingly, there is interest in building ethics-focused methods and tools to support the work of computing students and practitioners. Particularly in the HCI and Science, Technology, and Society (STS) literature, several frameworks and methodologies have addressed how values, critical reflections and ethics might be incorporated into designers' everyday work, including conceptual, theoretical, and methodological framings such as Value-Sensitive Design (VSD; [27-29]), critical design [7, 8], professional codes of conduct [59], Values at Play [25], value levers [52], steps to become an 'ethicist' [57], and others. According to a recent survey of 63 ethics-focused methods by Chivukula et al. [16], a wide range of methods and supports exist at varying levels of abstraction, including conceptual frames, theoretical commitments, and methods. In particular, methods represent a starting point for supporting pragmatic action, supporting designers to engage more deeply in various aspects of ethical complexity. While we do not explicitly address methods as supports for ethical behavior in the present study, we frame these existing approaches as potential mediators or interruptors for the problematic and "dark" behaviors that emerged in our study findings. 


\subsection{Criticality and "Dark" Design}

In the last decade, computing professionals have been increasingly interested in describing problematic behaviors that relate to persuasion, manipulation, and coercion-part of a broader critical "turn" [40] that has foregrounded social responsibility, social justice, and design justice $[18,20]$. One common term that has surged in the popular press to describe a class of problematic behaviors is "dark patterns," a neologism coined by practitioner and cognitive scientist Harry Brignull in 2010 that describes an element of "a user interface that has been carefully crafted to trick users into doing things" [2] using knowledge of human behavior and psychology against the user. Gray and colleagues [38] have built upon this definition to describe instances "where user value is supplanted in favor of shareholder value," articulating a range of strategies that designers take on to manipulate users which include nagging, obstruction, sneaking, interface interference, and forced action. Increasingly, dark design and dark patterns are being referenced in regulation and enforcement, as well as in the computing and technology communities $[4,5]$. Other relevant work in this space has addressed the need for "white hat" practices to fight back "dark UX" [22], the role of everyday citizens in discussing and raising issues of concern on social media as the work of "asshole designers" [17, 37], and the role of "bright" or "light" patterns that might be used instead of "dark" patterns [34]. All of this work relating to "dark" design points to instances where manipulative intent is hidden and disguised, leaving many consumers both unaware and unable to fight back [44, 47]. We leverage this interest and enhanced description of "dark" design practices and strategies in this paper as a means of better connecting the roles we identified in our protocol data to known dark patterns strategy types (see the Discussion section for this synthesis).

\section{METHOD}

We have taken a lab protocol approach [33] to describe the roles taken on by student designers as they worked to address a design task. Lab protocols have been widely used in psychology studies, but our connection to this method is primarily through design lab protocol studies-a common means of engaging with the complexity of design activities, allowing for both rich data capture and a controlled environment to compare experiences across protocol sessions [33]. Our main analytic focus in this paper includes how computing students engaged with ethical decision-making, which we represent based on the performative roles they took on to address the design task. The lab-based approach allowed us to simulate and control a scenario that replicates portions of a professional setting, which due to the controlled environmental variables and our ability to collect detailed data, facilitated the capture of cognitive moves that occur in design practice. Our lab protocol study included four groups of three computing students each, tracking them as they worked to address a real-world, ethically-nuanced design task in a single one hour session. The study was approved by our institutional IRB, and was constructed as a "deception study." Participants enrolled in the study were initially told that we were investigating their design decision-making behaviors, with no reference made to ethics or dark patterns. After the session was complete, we debriefed participants on the true purpose of the study-including its focus on ethics-and participants had the option to withdraw their participation at that time. All participants consented both at the beginning and during the debrief. Based on the data we collected, we analyzed participants' interactions to describe the various roles they took on as they made sense of the design situation and made concrete decisions to address the project brief. We describe these roles based on conversational turns, generated solutions, and post-hoc rationale from participants that supported a design move. We answer the following research question: What are the value-oriented roles taken on by computing students as they address an ethically-nuanced design task as a group?

\subsection{Participants}

Twelve participants participated in our lab protocol sessions, all of whom were undergraduate students from User Experience (UX) design and Industrial Engineering (IE) programs at a large Midwestern US university. These students represent common target areas of computing education, and all students had previously taken courses with an human-computer interaction (HCI) or human factors focus as part of their degree requirements. Each of these degree programs included coverage of professional ethics as required by ABET accreditation; IE included at least one course with a focus on ethics, and UX included ethics training integrated across multiple courses in the curriculum.

We recruited participants to form a stratified sample [49] by disciplinary background through professional connections and departmental listservs. We were seeking students who had previously completed design-related projects or had taken an HCI or human factors course. We grouped the participants to create two sessions (Session 1 and 4) with a mixture of UX and IE students and two sessions (Session 2 and 3) that had only UX or IE students. This allowed us to capture interactions among students from both disciplinary backgrounds, although we do not seek to conclusively compare disciplinary background as an aim of this paper.

\subsection{Lab Protocol Design}

We conducted lab protocol sessions with four groups of three student designers each, observing and video-recording each session to document participant interactions as they addressed an ethicallynuanced design task. Each protocol session duration was 60 minutes, divided into the following section: introduction to the process and design task (5 minutes), addressing the design task and generating solutions (45 minutes), and presenting the final outcomes and answering researcher questions (10 minutes). The student designers were given a design brief that included an explicitly manipulative design aim, with the stated goal of improving Amazon Alexa products. The researchers asked the participants to imagine they were working at Amazon when addressing the brief: "We at Amazon are working on improving our Amazon Alexa products. In order to do this, we need to collect more user data. We would like you to help us manipulate the user into giving up privacy permissions for their Amazon Alexa. We are hoping to gain the ability to listen in on all of the users' conversations and use this data to help advertisers better personalize the experience of using Amazon product." We framed the design task to replicate a real-world technology practice situation based on our prior research that had documented common ethical 
dilemmas in practice, where business stakeholders frequently approach designers and technologists with design goals that often prioritize business values over user values.

After introducing the design task, the participants were asked to generate one or more appropriate solutions (e.g., screen layout/wireframe, concepts, user task flow) to achieve the given design goal. The participants were provided with screenshots of the Alexa mobile app for their reference (Landing Page, Details Page, Privacy Page), and were free to alter or replace any of the elements on the screens as they felt necessary. Participants were also provided with sketching tools, paper, whiteboards, and markers for brainstorming and iteration. Additionally, the participants were provided with a flyer (Figure 1) consisting of interaction design principles such as visibility, feedback, affordance, mapping, constraint, consistency, learnability, and usability [48] and persuasive principles such as persistence, reduction, suggestion, prominence, exclusivity, and tunneling [26]. These principles were provided to mirror common persuasive technology principles leveraged in industry environments, and were described using a neutral language to avoid the framing of these principles as inherently "evil."

\subsection{Data Collection}

The protocol sessions were video and audio recorded with cameras positioned above the participants and from the front, capturing gestures, interaction with design artifacts, and movement during the session. These recordings were transcribed using an online transcription tool. The transcripts produced were then cleaned to add participant pseudonyms, timestamps, and inaudible instances. These transcripts were further used for data analysis along with the video recordings to decode the interactions between the designers. We also collected their sketches and whiteboard discussions for future reference alongside their conversational turns.

\subsection{Data Analysis}

We analyzed the transcripts through three rounds of iterative and reflexive thematic analysis [11]. In the first round, three researchers individually open coded [10] the different roles designers took on as they addressed the design task using one protocol transcript. This transcript was chosen as it was very engaging and gave rich insights into the designer's interactions. The three researchers carefully read each conversational turn with the goal of describing the value orientation(s) of the designer(s) and the role that these values had in informing the decision making process. All researchers were trained in conducting qualitative data collection and analysis through coursework and other research projects. After open coding individually, we came together along with the principal investigator to discuss the open codes. The various roles taken by the participants related to design cognition, design process, design production, value orientations, and decision making. The valueoriented moves depicted the designer's intentions to either orient towards the business stakeholder and business goals or user values and user-centered experiences. The majority of these roles reinforced the "dark"-yet-realistic design task and demonstrated a clear and considered effort to manipulate the user through their design solutions, although it became evident that some participants were also aware that they were dealing with "dark UX" practices. To focus and answer our research question, we jointly and reflexively created a candidate list of roles that related directly to the value orientations of the designers, setting aside design process moves such as: the director/planner, interface builder, consensus builder, or documenter.

In the second round of analysis, we focused more deeply on the value-oriented moves, observing two categories of roles as the participants expressed their value-related intentions: solutionfocused roles and rationale-focused roles. Solution-focused roles define the value-oriented roles that illustrate proposed or generated solutions in representationally-focused forms, as listed in Table 1. Rationale-focused roles define the value-oriented roles that relate to the student's intentions or the rationale and framing of the generated solutions, as listed in Table 2. Through further thematic coding of the transcripts, we built upon and revised the codebook of various roles as presented in Tables 1 and 2.

In the third round of analysis, each conversational turn from the four transcripts were exclusively coded based on the codebook using QSR NVivo. During this round of coding, we also introduced new codes that related to "research and design logistics," "design dialogues," and "communicator". These codes helped us to distinguish design discussions (previously described as "design process moves") such as sketching, planning and whiteboarding from valueoriented decision making. We observed that designers used real world scenarios to communicate their ideas and represent user task flows which did not directly include any value orientations, but were nevertheless an important part of the discourse. These instances were coded as "communicator" speech acts. For example, "The last one-once they kinda agree to share their privacy with Alexa, it might be better to have some small button here, where [they] can just [...] easily change, on and off button for privacy." In this example, the designer is detailing the user interface that includes a toggle button, with the discourse functioning as a communicator of their idea. This role was different from a rationale-focused role called "real-world scenario builder" because the latter role considers values included in the rationales provided as they manipulate the user. This explicit separation between design moves and value orientation moves aided us in aligning our results more towards our research question, keeping our focus on the value-oriented roles.

In the following sections, we present two different sets of roles that students took on as they addressed the design task (summarized in Tables 1 and 2). In Section 4, we describe a range of solutionfocused roles (the "puppeteer," the "nagger," and the "diluter") that demonstrate how ideas emerged and were refined. In Section 5, we describe a parallel range of rationale-focused roles (the "justifier" and the "capitalist") that demonstrate how teams justified and framed their design decisions.

\section{SOLUTION-FOCUSED ROLES}

In this section, we describe the solution focused roles taken on by participants, representing the solutions proposed and generated in their interactions and decision making. There are three broad roles under this category-the Puppeteer, Nagger, and Diluter. 


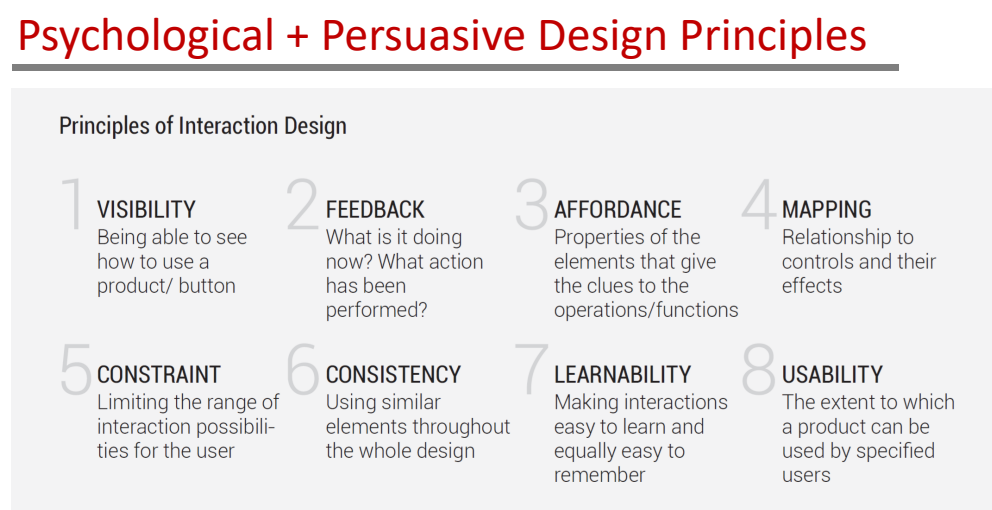

Practices That Persuade User's Actions
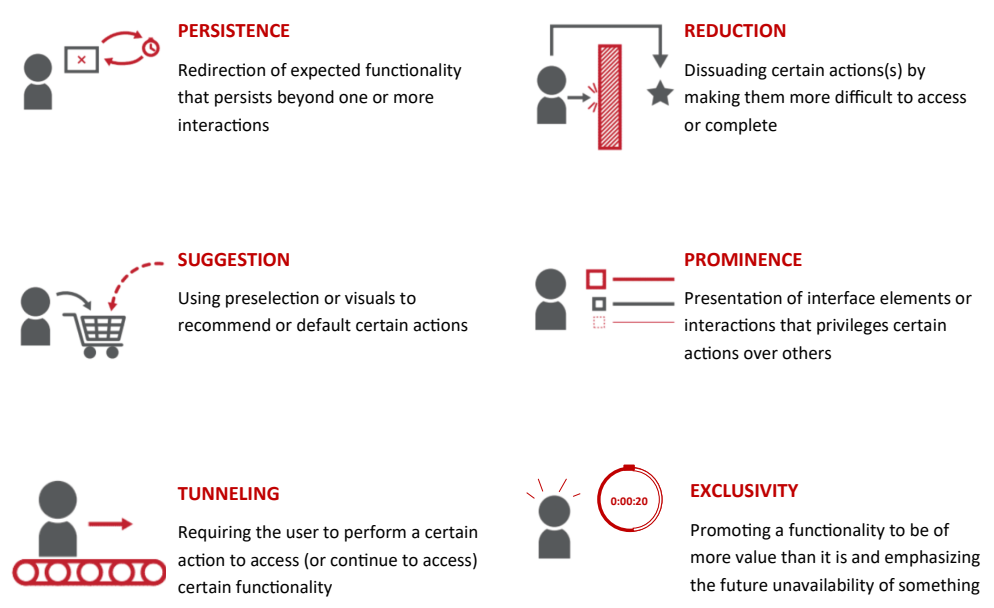

Figure 1: A neutrally-framed reference sheet provided to participants during the protocol study.

Table 1: Solution-Focused Roles.

\begin{tabular}{ll}
\hline Solution-focused Role & Description \\
\hline 1. Puppeteer & $\begin{array}{l}\text { Designs the task flow to encourage, steer, or nudge the user to follow a certain } \\
\text { path that benefits the stakeholder's goal. Types include: }\end{array}$ \\
$\begin{array}{l}\text { Designs a user task flow such that the user "can't get out of it," setting a trap for the } \\
\text { user that enables the stakeholder's goal. } \\
\text { Designs solutions to hide or bury information such that it is difficult for the user to find } \\
\text { it. } \\
\text { b. Camouflager }\end{array}$ & $\begin{array}{l}\text { Designs solutions to encourage the user to focus on "missing out" on features or func- } \\
\text { tionality. }\end{array}$ \\
c. FOMO-Dealer & Designs solutions that regularly interrupt or require the attention of the user. \\
\hline 2. Nagger & $\begin{array}{l}\text { Designs solutions that dilute the manipulative or persuasive nature of a solu- } \\
\text { tion by re-framing forms of manipulation instead of discarding them. }\end{array}$ \\
\hline 3. Diluter &
\end{tabular}


Table 2: Rationale-Focused Roles.

\begin{tabular}{|c|c|}
\hline $\begin{array}{l}\text { Rationale-focused } \\
\text { Role }\end{array}$ & Description \\
\hline 1. Justifier & $\begin{array}{l}\text { Rationalizes the generated solutions to support, illustrate, and realize both } \\
\text { stakeholder and design goals. Types include: }\end{array}$ \\
\hline $\begin{array}{l}\text { a. Real-world Scenario } \\
\text { Builder }\end{array}$ & $\begin{array}{l}\text { Rationalizes by comparing generated solutions and the user task flow using contextual } \\
\text { scenarios and positioning them in the real world to understand how it plays out. }\end{array}$ \\
\hline b. Empathy Manipulator & $\begin{array}{l}\text { Rationalizes proposed solutions by toying with a user's emotions to align with the } \\
\text { stakeholder's goal. }\end{array}$ \\
\hline c. Conscience & $\begin{array}{l}\text { Realizes manipulation in proposed solutions through reflection that is against their } \\
\text { internal values. }\end{array}$ \\
\hline d. Cynic & $\begin{array}{l}\text { Rationalizes manipulative facets of their decision making outcomes as the norm, with a } \\
\text { focus on reaching the stakeholders' goal. }\end{array}$ \\
\hline e. Contingency Planner & $\begin{array}{l}\text { Considers alternative scenarios and "what if" cases to design user task flows to reach } \\
\text { the stakeholder's goals. }\end{array}$ \\
\hline f. By the Book & $\begin{array}{l}\text { Follows and implements prescribed persuasive design principles to frame, create, or } \\
\text { rationalize their solutions. }\end{array}$ \\
\hline 2. Capitalist & $\begin{array}{l}\text { Rationalizes the proposed solutions through business objectives, advantage to } \\
\text { business stakeholders, and based on money, using the language of business } \\
\text { values to support their solutions. Types Include: }\end{array}$ \\
\hline a. Shareholder's Pet & $\begin{array}{l}\text { Rationalizes the solution as supporting the shareholder's business objectives with no } \\
\text { consideration of other stakeholder values. }\end{array}$ \\
\hline b. Business Analyst & $\begin{array}{l}\text { Rationalizes proposed solutions through examples of other business competitors, current } \\
\text { business trends, and examples of similar service or interface designs. }\end{array}$ \\
\hline
\end{tabular}

\subsection{Puppeteer}

A Puppeteer designs the task flow to encourage, steer, or nudge the user to follow a certain path that benefits the stakeholder's goal. As one participant described, the design outcomes need "to be created in a way such that the user doesn't feel-[...] the user should be sure that whatever he or she is doing is [...] perfectly alright". This sensibility aligns well with previous studies of dark patterns, wherein a designer uses their power to nudge users down a predetermined path through various levels of sneaking, obstruction, or forced action [38]. A puppeteer was observed in the following ways among the participants: Camouflager, Trap-Setter, and FOMODealer. Taking on one or more of these roles, the participant wanted to persuade the user to achieve the stakeholder's desires, even though it violated human-values [29] such as user consent, privacy, or ease of use.

4.1.1 Trap-Setter. A Trap-setter designs the user task flow to set a trap for the user to reach the stakeholder's goal so that the user "can't get out of it." In the process of carefully setting a trap informed by the stakeholder's goal, this designer fails to support common principles of interaction in the process. Trap-setters came up with solutions such as making certain steps in the task flow more difficult than they needed to be, pre-selecting the options for the user, interlinking features in order to access full functionality of the app, blocking features after usage to attract user's attention, and intentionally crashing the app upon selection of certain features. The most common feature proposed by multiple participants was to make it harder to get out of a certain task flow. If the users wanted to change the accepted permissions in the future, trap-setters decided to "make this difficult for them to find in the privacy setting." A group 1 participant articulated their approach this way: "Then, making the steps to get out of it so hard that they give up. So like, with the reduction [referring to the provided flyer of principles], the path they have to take to get out of it is just so much harder" and this resonates with a suggestion of a designer from group 3 saying "so the easiest-or like if we want users to feel to get into without knowing what they're doing so end up not wanting to get out of it, you want to make it as streamlined as possible for them, make it easy as possible for them to say yes and as hard as possible for them to say no." In both of these cases, the designers were intentionally setting a trap for the Amazon app user with an intent to streamline the process so that they have to "say yes" to the permissions. Trap-setters tried to interrupt the experience for those users who had not agreed to give microphone permissions by blocking certain features. For instance, a participant from group 2 proposed to present a pop-up while trying to use any feature saying "You can't use the full functionality. You can't use the voice control features without enabling your microphone." Participants also generated a solution to pre-select the agreement to "agree" as the users might feel I don't really care, I'll just go next. This would eventually make them agree to the permissions and "they're already screwed."

Participants also used trap-setting to block essential features, seeking to persuade the user to take certain actions by presenting the advantages of specific product features. For instance, if the user did not select the desired privacy permissions, the participant decided: "Fust don't give them access to the stuff that they will need at some point, like checking the weather." Here, the participants sought 
to trap the user in scenarios when they need access to features but could access them. As a part of this persuasive approach, participants suggested the use of advertising that could be supported by access to the user's microphone, with the proposed value of personalizing their experience if the product could listen to everything. For example, a group 3 participant described the design of a pop-up saying: "Allow Alexa to listen to you to give you better suggestions on songs, podcasts, sports, audiobooks."; similarly, a group 2 participant suggested including text that informed the users: "Hey, if you click this [permission] directly, you don't have to watch the ads."

4.1.2 Camouflager. A Camouflager designs solutions to hide or bury information such that it is difficult for the user to find or access it. This role is illustrated through an instance in which a participant concords with a solution of a fellow participant in their group by concluding: "So making sure that they don't discover this [privacy setting], basically." Taking on the role of a camouflager, participants proposed solutions such as using fine print or hidden meanings while asking for privacy permissions, bundling information with other features to divert the attention of the user from the voice feature, using repetition to build clicking habits that led to agreeing to the full access of microphone, and tweaking the information architecture of the app to hide the privacy features deep under the "Settings" section, making it difficult to access. A group 1 participant suggested that they wished to not explicitly specify the kind of permissions (i.e., privacy) the user woul dhave to give up to access Alexa's functionality, camouflaging the meaning of the sentences presented in the permission settings or agreement popups saying: "How about you just say 'Privacy permissions,' You don't specify what kind of, in this case audio right?.” A group 2 participant suggested: "you could bundle that and be like, 'Access your microphone and contacts." Through this solution, the participants were trying to combine permissions required for all features into a single sentence, encouraging the user to skip reading the different kinds of permissions requested. It is also seen that a camouflager was taking the role of a "trap-setter" through this solution by not giving a choice to the user to select specific permissions. Camouflagers also designed solutions that changed the appearance of pop-ups which requested permissions from the users. Participants from three different sessions came up with similar solutions relating to the arrangement of the pop-ups, sketching a solution that "ha[d] several pop-ups right behind each other" so they could manipulate the users with repetition of similar items and "having them click yes yes yes yes, [so] it becomes a habit." Another commonly proposed solution was to "hide" privacy permissions or settings in Alexa's app as expressed in this quote: "We'd put it like up in the setting but not just like in the settings-it's like in the settings somewhere awful." Through these examples, it is evident that the participants considered many different ways to bury or obscure the manipulation, working against common principles of interaction design relating to visibility and usability. Even when a participant from group 2 reflected, this tension was evident: "I know the IE standard is, like, you want everything very approachable and you can find everything very easily. I feel like in this case we are trying to not do that. So you would want to kind of bury the information a little bit."
4.1.3 FOMO-Dealer. A FOMO-Dealer designs solutions to encourage the user to focus on "missing out" on key features or functionality. In our given task, participants proposed solutions to make the user feel as if they are missing out certain features if they do not give full system access to the microphone. One participant described a framing of this goal as follows: "To make the full use out of your device, you would want to enable this." The participants taking on this role wanted to highlight what the users might be missing, communicating through e-mails and in-app information pop-ups. One such example included sending articles or newsletters via e-mail with subject line "How to get the most out of your Alexa device," with an intention to provide information to the users about the full use of their purchase as every user would be likely to avail themselves of when "they spend $X$ amount of dollars on it." Here, the participant tried to provide information that would encourage the user fear what they might be missing out on and letting them know "how easy it is to get access to whatever they're missing on their account" through one click of agreement to the microphone permissions.

\subsection{Nagger}

A Nagger designs solutions that regularly interrupt or otherwise require the attention of the user until they fall for a given manipulative target. Using this role, participants proposed solutions to nag the user with different kinds of in-app pop-ups and emails to persuade users to give full access to the microphone. Using inapp suggestions, participants proposed a variety of pop-ups if the user does not accept full access of the microphone. The functions of these pop-ups varied, including approaches such as continually reminding the users about giving full access to the microphone, interrupting the user interface with a prominent advertisement, and re-confirming the user's decision to disagree to the permissions. A group 1 participant suggested the design of a pop-up to regularly remind the user, describing a focus on: "prominence [referring to the provided flyer of principles], with this-making it pop up all the time and saying 'Hey, don't forget about this, it's awesome, peace out."' Another nagger suggested to make the need of full access to the microphone a "prominent part of the app-like how advertisements [...] pop up at the bottom constantly if you're not a premium member of it." Through this solution, the participant was proposing a large area of the app interface to advertise the microphone to the user constantly. Another way the participants used a nagging role was to regularly show a pop-up on top of the existing permissions pop-up asking the users "Are you sure want to say no to this?" when they disagree to give full access to the microphone, as an example of "confirm shaming." Using nagging outside of the app, participants also proposed how they could send emails to Alexa users, similar to how shoppers send offers to their customers during the holidays, saying: "So you could also push it through emails. I don't know if it is effective or not-probably not-but you can try. And it's all automated so, I mean, it's not an extra thing to go to, keep emailing them about it." Another participant extended this idea, describing how this could be automated and there is no extra effort required from the stakeholders to implement this solution. 


\subsection{Diluter}

A Diluter suggests solutions to dilute the manipulative or persuasive nature of a proposed solution by re-framing the form of manipulation instead of investigating or discarding it. This role was taken by participants when their "conscience" prompted them to critically reflect on the value orientation of the solutions generated, such as trapping the user by blocking features, camouflaging the permissions text, or nagging the user through pop-ups. In group 1, a participant proposed that the group block the features for trapping the user to encourage them give full access to the microphone, to which a fellow designer proposed: "[let's] limit them immensely," rather than completely blocking the features. Another fellow participant suggests that the app present a condition to the user saying "Oh, well, Alexa can only hear you up to five seconds because it can't record so it can't access that." Here, the participants sought to dilute the presentation of the trap, persuading the user to decide if they want to give full-access to unlock Alexa's full functionality. Group 3 participants often used phrases such as "sound nicer," "sound soft," or "occasionally softens the blow" as they were discussing ways to tweak the wording of the permission pop-up, with the goal of camouflaging the persuasive intent through the words used. In one example of this softening language, a group member said: "I don't like the access, 'cause that-we're really trying to soften the creepitivity with this." As a result of this decision, this group decided to dilute the manipulation of listening "continuously" to listening "occasionally," which still served the same ultimate purpose at the end in relation to the stakeholders goal. When participants diluted attempts at nagging the user, group 2 participants proposed to make the pop-ups less prominent by deciding to "pop it out, like, not constantly" as reminders for the users if they clicked on "later" for agreeing to the microphone access and decided to provide the settings as a pop-up the next time the user opens the app in a "week's" time.

\section{RATIONALE-FOCUSED ROLES}

In this section, we describe rationale focused roles taken on by participants as they engaged in deliberation regarding the intentions, rationale, and framing of the generated solutions. This set of roles positions the participants as discursive agents as they evaluate the rhetorical functioning of previous solution-focused conversations and rationalize why they relied upon various solution-focused roles. While taking on these roles, participants supported their concepts through design rationale, offering alternate considerations, framing the solution and problem space, and providing reasons why the different solutions might lead them to achieve the stakeholder's goal. There are two broad roles under this category-the fustifier and Capitalist.

\subsection{Justifier}

A Justifier rationalizes the generated solutions to support, illustrate, and realize stakeholder and design goals. The various types of justifications included: providing real world scenarios (Real-world Scenario Builder), considering ways to manipulate empathy (Empathy Manipulator), being cynical about the solutions (Cynic), questioning their conscience (The Conscience, identifying alternative plans to reach stakeholder's goal (Contingency Planner), and following prescribed persuasive principles as a means of user manipulation (By the Book).

5.1.1 Real-world Scenario Builder. A Real-world scenario builder rationalizes by envisioning proposed solutions and user task flows, as they might happen in a real world context. Using this role, participants externalized their thoughts and strengthened their rationale as they sought to convert abstract thoughts into concrete solutions by building on real-world scenarios or user behaviors. This role included strategies such as detailing the user scenario through task flow or personas, narrating the potential user interaction, visually representing the composition of the interface elements as it was viewed by the user, providing real-world scenarios or data to the users to manipulate their decision making, and rationalizing through potential, assumed, or anticipated user behaviors. For instance, by "playing out" the user task flow, a participant said: "So something like, initially when they download the app, asking them 'Would you like to be a part of our [... ] emailing list and then to receive access to like exclusive offers or stuff like that?' And then if they put in their email and say yes, I immediately contact them asking for this stuff?" In this example, the participant explicitly played out a scenario, envisioning the different interactions of the user before they accepted the desired privacy permissions. Participants provided explicit examples of scenarios or apps that would be connected to Alexa that they could block by being a trap-setter, including functionality such as contacts, voice calls, weather, and GPS positioning, which are essential for the user on a daily basis. Other ways participant used real-world scenarios included providing users with real information to persuade them to make a particular decision, as in this example: "So, you could say that from our research or whatever, from our data, X\% or X number of users have used this feature and observed an increase in productivity so it really helps you and kind of convince them." Here, the designer is proposing the presentation of usage data to show the increase in $X$ number of users' productivity. Speculating on user behaviors through potential real-world scenarios, participants also articulated key assumptions about their design space when a group 2 member said: "Yeah, I mean, think about this, if somebody downloads this Alexa app, so which means they already purchased either the speaker or any other Amazon app." This scenario connected the dots between previous rationale that the user already had the required equipment in place and had invested money to purchase (as illustrated in FOMO-Dealer), proposing that the user would likely agree to the permissions to unlock full functionality of the product. Overall, this role allowed the participants to justify a certain solution, thereby externalizing the designer's decision making process as they sought to convince fellow participants on how the solution may aid in reaching the stakeholder's goals.

5.1.2 Empathy Manipulator. An Empathy manipulator rationalizes proposed solutions by toying with user's emotions to align with the stakeholder's goal. While rationalizing their solutions, an empathy manipulator would say: "it's empathy-using their empathy to like manipulate them," and "using a user's emotion to get what you want." Participants provided rationale such as making users feel "really awful" by hiding privacy settings, creating a false sense of power, forcing the users through nagging, making them eager about the features they are missing, creating a fear of missing out on functionality, convincing the user by providing information through various 
mediums, creating confusion in user's decisions, toying with their weakness towards monetary values/ investments in the product, annoying or upsetting the user to trap into the designer's guided task flow, making the user more impatient through nagging, and presenting Alexa as more human to persuade the user's emotions. Group 4 participants sought to appeal to the user's emotions by making the interactions with Alexa more human as the current app design "seems a little robotic." Two other prominent ways of manipulating the user emotionally included making it hard for the users to interact with the settings in the app where the user would think "Whatever, I'll just give up" and creating a sense of missing out through information and nagging using in-app pop-ups and e-mails (as evident in solution-focused roles, nagger and FOMO). Along with these other means of manipulating emotions, blocking the features (as done by a Puppeteer) was also used to anticipate making the user annoyed or upset, encouraging the user to decide: "Yeah. I would just go into settings and let 'em use the microphone."

5.1.3 Conscience. A Conscience realizes that they are trying to do something against their internal values as they reflect on the given design brief, their proposed solutions, and rationale supporting their decision making process, often expressing their concerns when they realize they are being manipulative or persuasive. However, participants taking on this role increased awareness but did not stop them from persuading the user to achieve the stakeholder's intent because they were "hired to do so." This role was evident through different kinds of rationale-focused discussions, for instance, when a participant tried to express the "roughness" of the design brief, when a participant is "not okay" with the implemented solutions, or when a participant disagrees to a proposed solution as they would not have accepted it if had to experience the solution themselves. While reading the design brief, a group 3 participant called out these behaviors explicitly, revealing their awareness: "This is Dark UX." However, this recognition of a design strategy being "dark UX" did not stop the participant from creating manipulative solutions, and participants went ahead solving the design brief using the manipulative approach since the group thought: "it's interesting." We observed multiple instances of similar behaviors that related to the value tension of the conscience, such as a group 4 participant that reacted to a fellow designer's proposed solution saying "this is really weird, I don't like it. This is not okay"; a group 3 participant expresseing "I feel awful" when trying to re-word the permissions in a persuasive manner; a group 3 participant's conscience while reading out the given design brief said: "Manipulate the user. I don't know, I kind of feel bad doing this”; another participant describing the value trade-offs in their solutions, expressing "This is so dark, 'cause it goes-promoting a functionality to be of more value than it is. It's valuable to you a little bit, but at the same time they're listening to everything"; and a participant commenting about the listening feature of Alexa saying: "I find like reading that I'm like, oh, it's listening to me-that freaks me out." Through this role, some participants also expressed how they would not agree to permissions asked for full access of the microphone if they were the user. A group 3 participant reflected: "So, I don't think if we put a pop-up saying, 'Can we listen to part of your conversation?' I would say no, personally." This participant clearly expressed they would not want to experience their own manipulative techniques, but went ahead in order to support the stakeholder's goal by softening the wording of the permissions for the users to agree (being a Diluter). Similarly, participants reflected several possible real-world scenarios where Alexa could be listening to credit card details of the users, observing: "that's gonna be so sketchy." These instances exemplify how the participants critically reflected on the design brief, realizing it represented a manipulative design space. However, these reflections did not restrict their decision making in implementing these solutions or even accepting to engage in the design space without reframing.

5.1.4 Cynic. A Cynic rationalizes manipulative facets of their decision making as the norm, recognizing that these facets may serve as a means to reaching the stakeholder's goal. In our study, participants that took on the role of a cynic rationalized the use of pop-ups to camouflage microphone privacy permissions, actively relying upon building a habit of the user in clicking "Yes" multiple times before they reach the screen to give the privacy permissions. As a pattern of behavior, cynics also understood user behaviors relating to privacy, with knowledge that many consumers do not fully consider information privacy and impacts of privacy violation even when they are made aware of the impact of sharing these data. A group 3 designer rationalized this behavior as follows: " They've taught our generation, okay like, 'don't let people have your information,' but the way that the companies are doing it, we don't really realize" and "They don't care." This participant rationalized their proposed solutions-and the manipulation inherent in themas part of the status quo, recognizing that companies commonly manipulate the users in relation to information breaches, and using this recognition as leverage to incorporate similar solutions to achieve the stakeholder's goal. Another participant references this leveraging behavior and the designer's "dark" role in using this behavior against the consumer as follows: "Cause if you keep giving people options, they'll start thinking more and more about it, like something, their privacy being taken away." Another user behavior recognized by a cynic was how they felt users are usually "neutral" during on-boarding a new app and want that process to be done "quickly." Using this knowledge, a cynic takes advantage of this situation by rationalizing the pop-up solution by saying "Okay, and they go yes, it's quick, it's fast for them, they don't really know, they're still in the dark I guess. User kept in dark and move on." Here, the designer accepted that the user would not pay much attention to the privacy permissions, and building on this knowledge and lack of consumer attention, they could use other dark strategies to get full access to the microphone as stated in the stakeholder's goal.

5.1.5 Contingency Planner. A Contingency planner considers and thinks through alternative scenarios and "what if" cases to design user task flows to reach the stakeholder's goals. Participants taking on this role used scenario-based rationalization by identifying and considering alternative design spaces that might restrict them from achieving the stakeholder's goal, constantly thinking about "if this does not happen" situations to create back-up solutions through a range of solution-focused roles. Contingency planners continuously reflected on when and how solutions created through their solutionfocused roles might fail, and with what impact, including some of the following contingencies: What if the user does not fall for the 
trap to agree to the permissions (designed by a Trap-setter); What if the user disagrees to the permissions even after camouflaging? (designed by a Camouflager); What if a user opts not to agree to the permissions even after repeated attempts? (designed by a Nagger); or What if the user doesn't care about missing out on Alexa's full functionality? (designed by a FOMO-Dealer). A group 1 participant considered a scenario when the user does not grant permissions, reflecting: "What if this [agreeing to the permissions] is like 'No.' And then you just lose them, like you can never like regain access to them" or "but what do we do if they say no from the start and they go despite how we make them go through so much to get out of it." In another example, a group 2 participant questioned what would happen if the user clicked "later" on the permissions pop-up, to which a fellow participant suggested "When you say later, then you're not gonna have full functionality of the device." Here, the first designer took the role of being a contingency planner and the latter supported the thought by being a trap-setter.

5.1.6 By the Book. A By the Book designer follows and implements prescribed persuasive design principles in dark ways to frame, create, or rationalize their solutions. Throughout all of the sessions, we observed the participants referring to the "Persuasive Principles" flyer given to them along with other materials as a priming tool. Many participants were very explicit regarding their use of these principles as an aid to reach the stakeholder's goal, mirroring real-world access to growth hacking resources or persuasive design principles (such as the ones from Fogg we provided). For example, a group 3 participant suggested: "Yeah, do you guys just wanna go through [the flyer], because I feel like it might help" and a group 1 designer mentioned: "Specifically to listen in on certain conversations and such. So, uh, we could use some of these psychological and persuasive design principles to kind get to that [design brief]." Group 2 participants attempted to combine two or more of these principles to generate solutions, to which a fellow participant responded by saying "I like the idea of using more than one of those principles, so it's, like, more persuasive." The principles of exclusivity, tunnelling, reduction, and prominence were frequently used by participants to generate and rationalize solutions. Reduction was used by a trap-setter to rationalize solutions such as making it more difficult for the user to change privacy settings or not giving the users the toggle to turn off the privacy settings. Prominence was used to rationalize nagging the user through pop-ups, highlighting the features that the user might miss if they do not agree to the permissions. Through nagging, this principle was used in conjunction with persistence to justify regularly showing the user messages the in-app pop-ups. Tunnelling was used to rationalize the restriction of features for users who do not agree to the permissions. These above examples demonstrate how the participants implemented the persuasive principles as a bridge between rationalization and solution generation, using these principles as a "dark" checklist for reaching the stakeholder's goal rather than as a means of considering a balance of user and stakeholder needs.

\subsection{Capitalist}

A Capitalist rationalizes their proposed solutions through business objectives, advantage to business stakeholders, and based on money, often using the language of business values to support their solutions. The Capitalist provided business-centric rationale, distinguished from rationale provided by a fustifier, which are more user-centric. We observed that the participants, by taking on the role of a Justifier or a Capitalist, supported the stakeholder through their intentions, but the discursive functions of these roles built on user scenarios in the former and stakeholder scenarios in the latter. The two kinds of Capitalist roles include the Shareholder's Pet and the Business Analyst.

5.2.1 Shareholder's Pet. A Shareholder's Pet rationalizes the solution as supporting the shareholder's business objectives with no consideration of other stakeholder values or needs. Using this role, participants focused their attention primarily on the stakeholder, as illustrated by a group 2 participant: "I mean, yeah, if we use ads, we can get more money. [laughs]" and a group 1 participant: "Amazon doesn't get what they want." Stakeholder's Pet rationale included instances when participants accepted that users did not (or should not) have a choice in the service provided by the business, scenarios where participants tweaked the solutions when they realized that the shareholder's business was at stake, decisions where the proposed solutions would be an advantageous for the business, and overall, instances where the participants were responding affirmatively to the values embedded in the design brief which explicitly asked the participants to "manipulate the user." The task given to the participants requested users to be manipulated into gaining full access to the microphone, which in itself is a task that primarily supports the shareholders and frames users as pawns. We recorded instances where the participants agreed to solving the task as it was framed, even though they had the ability to reject or reframe the brief.

5.2.2 Business Analyst. A Business Analyst rationalizes proposed solutions through examples of other business competitors, current business trends, and examples of similar service or interface designs. Within a capitalistic mindset, participants acting as business analysts leveraged similar solutions from existing services, rationalizing their solutions as similar to existing solutions. In one example, a group 1 designer noted how Apple gets around the permissions for access of contacts or makes it hard for the user to unsubscribe, concluding: "And that's how they make their money." Other groups referred to Samsung Mobile Phone or iPhone's interface, changing their "Settings" page to change the information architecture on every update; leveraged Youtube's survey to improve personalization to rationalize the use of pop-ups that mentioned that the experience would be tailored according to user's choice if they agreed to the microphone access; and built upon Macy's email subscription where they send e-mails about "Fall offers" and examples of user agreement on gaming apps where the user is not allowed to go past a certain step if the permissions are not accepted. Along with examples from competitors, participants also leveraged Amazon's existing services beyond Alexa, rationalizing how the company could provide users with full functionality of free Prime accounts and Amazon Music with the collected data through privacy permissions, further trapping the user within the Amazon system. These examples could have been used to critically reflect on the user's experience and address potential value tensions. Instead, the Business Analyst role was used by the team to instead replicate and leverage similar forms of persuasion in their own solutions. Overall, this role 
relied upon " that's how they like, run their business" as a rationale for their design solutions and the participants frequently used this role to replicate successful persuasive business strategies.

\section{DISCUSSION}

Through our analysis, we have described the various roles taken on by computing students as they navigated an ethically-nuanced design space. These roles enabled the participants to engage with both solutions and design rationales, often with "dark " intentions of persuading or manipulating users in order to support business goals. In this section, we first draw connections across the analyzed roles and dark patterns, positioning the interactivity of these roles as a means of describing both the ethical engagement of computing students and practitioners, and the links between ethical awareness, engagement, and social responsibility. Second, we present implementable suggestions to improve ethical awareness and engagement in computing education, with the goal of enhancing students' pragmatic awareness of ethical design processes and complexities in decision making.

\subsection{Interactive Roles Can Perpetuate "Dark" Design and Limit Ethically-Focused Action}

Somewhat surprisingly, we observed that the sensitivity of students towards user and human values did not necessarily impact their decisions or ethically-focused outcomes. In many cases, values were acknowledged, only then to be leveraged in persuading, manipulating, or coercing users towards stakeholder-directed outcomes (illustrated by Conscience), activating potentially dark intentions on the part of the designer. Through the analyzed roles, we see a strong relationship between participants' utilization of the persuasive principles formulated by Fogg [26] and characterizations of dark patterns strategies indicated previously by Gray et al. [38] (i.e., nagging, obstruction, sneaking, interface interference, and forced action). Rather than investigating how Fogg's persuasive principles might impact the user or society in negative ways, participants instead used the persuasive principles as a veritable "playbook" to manipulate users in ways that addressed the design brief. For example, solution-focused roles like Nagger relied upon the dark pattern strategy "nagging" defined as "a repeated intrusion during normal interaction, where the user's desired task is interrupted one or more times by other tasks not directly related to the one the user is focusing on" [38] when asking users to give full access to the microphone. Trap-Setters used the dark pattern strategy "forced action," defined as "any situation in which users are required to perform a specific action to access (or continue to access) specific functionality" [38], as they were bundling various services together to agree to privacy permissions without giving users a choice to select. Through these instances, among others, participants appeared to operationalize certain dark pattern strategies into the proposed solutions to reach the stakeholder's goal, frequently leveraging persuasive principles as a vehicle. By the Book created a design space that had this "dark" potential, where participants used principles that were positively framed in the provided flyer to persuade users without any reflection on how these actions might negatively impact the user experience. There were also important areas of interplay among roles as it related to value awareness. The Business
Analyst validated their persuasive solutions since they were already being implemented in other technological products to fulfill the stakeholder's goal, while the Conscience demanded not to implement these solutions in their "darkest" form, leaving the Diluter to proceed with slightly weaker forms of the same manipulative, persuasive, or coercive design outcomes that supported business values. The individual designers had all the appropriate knowledge and awareness regarding the kinds of manipulation and impacts on the user, yet instead of critically evaluating and speculating about the ethical concerns, their goal remained solely to "manipulate the user" as the design task requested. To some degree, this kind of design action that prioritized business values unquestioningly could be anticipated based on other studies, such as the Milgram experiment-where participants engaged in harmful conduct towards other human beings due in part to perceptions of obedience to authority-however, the degree to which participants navigated value awareness, manipulation, and dilution to appear to address business goals while not looking totally "evil" represented more nuance relating to personal responsibility, design action, and potential social impact than we might have expected.

The persuasive and manipulative roles taken on by the students to generate solutions and rationalize their decision making took on properties of propagation and dynamic interaction. During interactions among the designers in their groups, the range of roles were not linked to a particular participant, but rather pervasive, dynamic, and persistent among the group of participants as they worked together to manipulate the user and support the stakeholder's goal. We have detailed these propagation interactions among team members, including the team dynamics and individual roles that led to successful propagation, in a parallel analysis which we report in Chivukula and Gray [15]. Even given the disciplinary differences of the students-with differing training on ethics and social responsibility in IE and UX-we saw no particular differences in the emergence and movement among disciplinary perspectives in our analysis. We found the patterns of propagation that introduced and then sustained manipulative intent to also rely upon dynamic and emergent relationships among the solution-focused and rationalefocused roles in ways that informed both the emergence of ethical awareness and the dilution of manipulative outcomes that built a "dark" consensus among group members. Overall, individually or as a group, these findings about the participants' behaviors provides a descriptive account of how computing students react to a given stakeholder request and the interactions capture new dimensions of ethical engagement that may not be as readily explored through other common ethics education techniques such as case studies. This interaction among the roles illustrates the nuanced and diverse roles taken by these participants that propagated "dark" intentions that led to ethically problematic design outcomes. The behaviors we observed among the participants as they were deliberating and accepting the manipulative design brief provides insight into how educators might better support both awareness and pragmatic tools for action that aid in ethically-focused and value-conscious decision making. As much as participants did demonstrate levels of awareness of their ethical responsibility towards their users, we question the participants' perception of their felt agency to work against or actively reject the manipulative design goal. The students potentially had the space to confront the darkness of the design space in 
ways that they might not in a formal industry setting where their employment might be at risk, but instead the participants proposed a range of solutions taking on a complex set of manipulativelyfocused roles. This points towards questions for future work, such as: How do computing students identify their agency in relation to a design decision or design space? How do computing students identify their responsibility towards users or society in relation to their proposed solution? What are some ways in which students can be supported to critically reflect on their ethical responsibility and role?

\subsection{Pedagogical Opportunities to Support Ethical Awareness and Action}

Given the descriptive accounts of computing students' behaviors, we have identified opportunities for pedagogical interventions that may enhance students' pragmatic awareness of ethical design processes and complexities in decision making. Through the construction of this protocol study, we intentionally sought to frame the design brief in ways that foreground key business tensions commonly present in industry work. As revealed in our findings, this ethicallyproblematic framing of design outcomes reveals attitudes to ethical engagement that are not focused on awareness or even disciplinary prerogative, but rather on pragmatic engagement both with ethical awareness and links to socially responsible design outcomes. Building on these findings, we offer suggestions to strengthen computing education experiences that relate to pragmatic reflection, tool reflection, and self reflection.

From a pragmatic reflection perspective, we propose the need for more ecologically-resonant ways for students engage with ethical concerns in their course experiences, and when appropriate, build the capacity to question authority. While methodologies such as VSD provide entry points towards building ethical awareness, there is less support for building ethically-focused roles with which students and practitioners can constructively engage with ethical dilemmas, tensions, or responsibilities towards the user or society. As demonstrated in our lab protocol outcomes, real world design challenges demonstrate the benefit of raising ethical complexity in ways that are increasingly being taken up by computing educators (e.g., using examples of discrimination when teaching about algorithms). An additional set of "on the ground" instructional techniques to engage computing students in grappling with the ethical complexities that arise during decision making-including the ability to reject the initial framing of the design situation-may increase the ability of students to engage in pragmatic reflection, both in the moment and over time. In particular, we propose instructional techniques that are aligned with multiple frames of ethical reasoning, including practical business, disciplinary, user, and societal factors. Each of these frames foregrounds particular forms of what Gray and Chivukula [36] have described as "ethical design complexity." Using this pragmatic framing technique, students may be able to better consider the ethical complexities that are present in everyday work scenarios, building on existing professional code of ethics alongside pragmatic roles that are taken on to strengthen or weaken ethical commitments. We suggest bringing ecological or organizational dimensions of computing practice into the classroom, use ethical dilemmas faced by practitioners to stimulate conversations around ethical responsibility, and prepare students for practical barriers in applied ethics which frequently involve people in different disciplinary roles with varying levels of power, attendance to a range of stakeholder objectives, and felt levels of personal and disciplinary responsibility towards product outcomes. By using a pragmatic frame to engage with ethics, computing students may be better positioned to build awareness and advocacy for both users and ethically-focused societal outcomes.

From a tool reflection perspective, we propose a pedagogical focus on the intentions that designers bring to design situations, focusing on how these intentions may link to the selection of appropriate tools to support ethical action. The process of method selection and use is poorly understood in both design education and practice contexts, and we have found this area in particular to be a productive space to identify intentions that drive tool selection, and map these intentions in relation to matters of ethical concern. Recent work has begun to articulate some of the ethics-focused supports that may build student and practitioner awareness or capacity to act [16], but the felt need of students for such methods or tools is an important area for future study.

As part of this future work, computing education researchers may consider the role of particular "intentions" to describe a set of expectations a student might have about knowledge of their ethical standpoint and articulate a particular scope of design complexity that a designer might be experiencing. For instance, intentions such as wanting to "break the design" or find flaws, wanting to identify a set of guiding values for a given project, or wanting to align team members in addressing difficult scenarios or contexts frame both ethical awareness and the need for ethical support. By framing these intentions around particular areas of project-based, team-based, or personal engagements with issues of ethical concern, we can create awareness among computing students with the potential for action.

From a self reflection perspective, computing students can be engaged in self-reflection - in the moment and overall - about their roles in decision making and realize their ethical responsibility. The list of roles described in this paper can act as critical reflection tools for designers to become aware of various "hats" they take on during decision making, knowingly or unknowingly. From our observations regarding the interactive nature of these roles among a group of students, the list of solution-focused and rationale-focused roles may aid students in realizing that ethical awareness and action require continuous balance that begins-but is not fully satisfied-with the knowledge that a behavior or outcome is potentially problematic. Through self-reflection, each student may find ways to better articulate the values and philosophy that guides their work, while also encouraging the articulation of these values as they engage in student and practitioner teams.

\section{CONCLUSION}

In this paper, we present the results of a role analysis that provides a descriptive account of ethical reasoning that arises among computing students as they designed for a multi-channel voice user interface (VUI) experience. We describe a set of solution-focused and rationale-focused "dark" design roles taken on by these students, which as a set elucidate the discursive means through which these students engaged with and sought to balance manipulative 
and value-centered intentions. These roles were often actively propagated through design discourses, impacting the other designers in ethically-negative ways. Even when students were aware of ethical concerns based on their educational training, this awareness did not consistently result in ethically-sound decisions, underscoring the need for more pragmatically-focused ethical training in computing education. Based on these findings, we propose implementable pedagogical interventions in computing education from a pragmatic, tool, and self-reflection perspectives.

\section{ACKNOWLEDGMENTS}

This work is funded in part by the National Science Foundation under Grant Nos. 1657310 and 1909714.

\section{REFERENCES}

[1] [n.d.]. ABET Accreditation. https://www.abet.org/. Accessed: 2021-2-16.

[2] 2014. Dark patterns: User interfaces designed to trick people. http://talks.uipatterns.com/videos/dark-patterns-user-interfaces-designed-to-trick-people. http://talks.ui-patterns.com/videos/dark-patterns-user-interfaces-designed-totrick-people Accessed: 2021-3-26.

[3] 2017. GenderMag Teach. https://sites.google.com/site/gendermagteach/. Accessed: 2020-12-30.

[4] 2020. "Dark patterns" make their appearance in California's new privacy law. https://www.jdsupra.com/legalnews/dark-patterns-make-their-appearancein-30570/. https://www.jdsupra.com/legalnews/dark-patterns-make-theirappearance-in-30570/ Accessed: 2021-1-4

[5] 2020. Dark Patterns: Regulating Digital Design. https://www.stiftung-nv.de/ en/publication/dark-patterns-regulating-digital-design. https://www.stiftungnv.de/en/publication/dark-patterns-regulating-digital-design Accessed: 2020-8 2.

[6] Janet S Adams, Armen Tashchian, and Ted H Shore. 2001. Codes of ethics as signals for ethical behavior. Fournal of business ethics: $7 B E 29,3$ (2001) 199-211. https://idp.springer.com/authorize/casa?redirect_uri=https //link.springer.com/article/10.1023/A:1026576421399\&casa token= pde4sKpjVKUAAAAA:u9yumz0xz6Zbf66tZ7ESxpNOmc_BM51Dp2TAiXoovTBBWxm4ntzWUV4FG5eKbeyz0LFhF2tWs2IOlCe

[7] Jeffrey Bardzell. 2014. Critical and cultural approaches to HCI. The SAGE handbook of digital technology research (2014), 130-143.

[8] Jeffrey Bardzell and Shaowen Bardzell. 2013. What is critical about critical design? In Proceedings of the SIGCHI conference on human factors in computing systems. 3297-3306.

[9] Jane Blanken-Webb, Imani Palmer, Nicholas C Burbules, Roy H Campbell, and Masooda Bashir. 2018. A Case Study-based Cybersecurity Ethics Curriculum. In 2018 \{USENIX\} Workshop on Advances in Security Education (\{ASE\} 18). usenix.org.

[10] Virginia Braun and Victoria Clarke. 2006. Using thematic analysis in psychology. Qual. Res. Psychol. 3, 2 (Jan. 2006), 77-101. https://doi.org/10.1191/ 1478088706qp063oa

[11] Virginia Braun and Victoria Clarke. 2019. Reflecting on reflexive thematic analysis Qualitative Research in Sport, Exercise and Health 11, 4 (Aug. 2019), 589-597. https://doi.org/10.1080/2159676X.2019.1628806

[12] Bo Brinkman and Keith W Miller. 2017. The Code of Ethics Quiz Show. In Proceedings of the 2017 ACM SIGCSE Technical Symposium on Computer Science Education (Seattle, Washington, USA) (SIGCSE '17). Association for Computing Machinery, New York, NY, USA, 679-680. https://doi.org/10.1145/3017680.3017803

[13] Ryan C Campbell, Ken Yasuhara, and Denise Wilson. 2015. Ethics in Engineering Students' Design Considerations: Case Studies of Electric Power Systems for the "Developing World". In Proceedings of the American Society for Engineering Education.

[14] Jeffrey K H Chan. 2018. Design ethics: Reflecting on the ethical dimensions of technology, sustainability, and responsibility in the Anthropocene. Design Studies 54 (Jan. 2018), 184-200. https://doi.org/10.1016/j.destud.2017.09.005

[15] Shruthi Sai Chivukula and Colin M Gray. 2020. Co-Evolving Towards Evil Design Outcomes: Mapping Problem and Solution Process Moves. In DRS Biennial Conference Series (Brisbane, Australia). Design Research Society. https://doi.org/ $10.21606 /$ drs. 2020.107

[16] Shruthi Sai Chivukula, Ziqing Li, Anne C. Pivonka, Jingning Chen, and Colin M. Gray. 2021. Surveying the Landscape of Ethics-Focused Design Methods. arXiv:2102.08909 [cs.HC]

[17] Shruthi S Chivukula, Chris Watkins, Lucca McKay, and Colin M Gray. 2019 "Nothing Comes Before Profit": Asshole Design in the Wild. In CHI EA '19: CHI'19 Extended Abstracts on Human Factors in Computing Systems. LBW1314. https: //doi.org/10.1145/3290607.3312863
[18] Sasha Costanza-Chock. 2020. Design fustice: Community-Led Practices to Build the Worlds We Need. MIT Press. https://play.google.com/store/books/details? id=h4LPDwAAQBAJ

[19] Mary L Cummings. 2006. Integrating ethics in design through the value-sensitive design approach. Science and engineering ethics 12, 4 (Oct. 2006), 701-715. https: //www.ncbi.nlm.nih.gov/pubmed/17199145

[20] Lynn Dombrowski, Ellie Harmon, and Sarah Fox. 2016. Social Justice-Oriented Interaction Design: Outlining Key Design Strategies and Commitments. In Proceedings of the 2016 ACM Conference on Designing Interactive Systems (Brisbane, QLD, Australia). ACM, New York, New York, USA, 656-671. https: //doi.org/10.1145/2901790.2901861

[21] Stacy A Doore, Casey Fiesler, Michael S Kirkpatrick, Evan Peck, and Mehran Sahami. 2020. Assignments that Blend Ethics and Technology. In Proceedings of the 51st ACM Technical Symposium on Computer Science Education (Portland, OR, USA) (SIGCSE '20). Association for Computing Machinery, New York, NY, USA, 475-476. https://doi.org/10.1145/3328778.3366994

[22] Trine Falbe, Kim Andersen, and Martin Michael Frederiksen. 2017. White Hat UX. Smashing Media AG. https://play.google.com/store/books/details?id= b0EiygEACAAJ

[23] Casey Fiesler, Mikhaila Friske, Natalie Garrett, Felix Muzny, Jessie J Smith, and Jason Zietz. 2021. Integrating Ethics into Introductory Programming Classes. In Proceedings of the 52nd ACM Technical Symposium on Computer Science Education (SIGCSE'21). New York, NY, USA: ACM. https://cmci.colorado.edu/ cafi5706/ SIGCSE2021_IntegratingEthics.pdf

[24] Casey Fiesler, Natalie Garrett, and Nathan Beard. 2020. What Do We Teach When We Teach Tech Ethics? A Syllabi Analysis. In Proceedings of the 51st ACM Technical Symposium on Computer Science Education (Portland, OR, USA) (SIGCSE '20). Association for Computing Machinery, New York, NY, USA, 289-295. https://doi.org/10.1145/3328778.3366825

[25] Mary Flanagan and Helen Nissenbaum. 2014. Values at Play in Digital Games. MIT Press. https://doi.org/10.1177/1461444816631742

[26] B J Fogg. 2009. Creating persuasive technologies: an eight-step design process. In Proceedings of the 4th International Conference on Persuasive Technology (Claremont, California). ACM, New York, New York, USA, 44. https: //doi.org/10.1145/1541948.1542005

[27] Batya Friedman and David G Hendry. 2019. Value Sensitive Design: Shaping Technology with Moral Imagination. MIT Press.

[28] Batya Friedman, Peter Kahn, and Alan Borning. 2002. Value sensitive design: Theory and methods. University of Washington technical report 02-12 (2002)

[29] Batya Friedman, Peter H Kahn, Alan Borning, and Alina Huldtgren. 2013. Value Sensitive Design and Information Systems. In Early engagement and new technologies: Opening up the laboratory, Neelke Doorn, Daan Schuurbiers, Ibo van de Poel, and Michael E Gorman (Eds.). Springer Netherlands, Dordrecht, 55-95. https://doi.org/10.1007/978-94-007-7844-3 4

[30] Ajit G. Pillai, A Baki Kocaballi, Tuck Wah Leong, Rafael A. Calvo, Nassim Parvin, Katie Shilton, Jenny Waycott, Casey Fiesler, John C. Havens, and Naseem Ahmadpour. 2021. Co-designing Resources for Ethics Education in HCI. In Extended Abstracts of the 2021 CHI Conference on Human Factors in Computing Systems. Association for Computing Machinery, New York, NY, USA, 1-5. https://doi.org/10.1145/3411763.3441349

[31] Natalie Garrett, Nathan Beard, and Casey Fiesler. 2020. More Than "If Time Allows" The Role of Ethics in AI Education. In Proceedings of the AAAI/ACM Conference on AI, Ethics, and Society. dl.acm.org, 272-278.

[32] Natalie Garrett, Mikhaila Friske, and Casey Fiesler. 2020. Ethics from the Start: Exploring Student Attitudes and Creating Interventions in Intro Programming Classes. In Proceedings of the 51st ACM Technical Symposium on Computer Science Education (Portland, OR, USA) (SIGCSE '20). Association for Computing Machinery, New York, NY, USA, 1348. https://doi.org/10.1145/3328778.3372638

[33] John S Gero and Thomas Mc Neill. 1998. An approach to the analysis of design protocols. Design Studies 19, 1 (Jan. 1998), 21-61. https://doi.org/10.1016/S0142694X(97)00015-X

[34] Paul Graßl, Hanna Schraffenberger, Frederik Zuiderveen Borgesius, and Moniek Buijzen. 2021. Dark and Bright Patterns in Cookie Consent Requests. , 38 pages. https://doi.org/10.33621/jdsr.v3i1.54

[35] Colin Gray, Seda Yilmaz, Shanna Daly, Colleen Seifert, and Richard Gonzalez. 2015. Idea generation through empathy: Reimagining the 'cognitive walkthrough'. In 2015 ASEE Annual Conference and Exposition Proceedings (Seattle, Washington) (Industrial Design Conference Presentations, Posters and Proceedings). ASEE Conferences. https://doi.org/10.18260/p.24208

[36] Colin M Gray and Shruthi Sai Chivukula. 2019. Ethical Mediation in UX Practice. In Proceedings of the 2019 CHI Conference on Human Factors in Computing Systems (Glasgow, Scotland Uk) (CHI '19). ACM, New York, NY, USA, 178:1-178:11. https: //doi.org/10.1145/3290605.3300408

[37] Colin M Gray and Shruthi Sai Chivukula. 2021. "That's dastardly ingenious": Ethical Argumentation Strategies on Reddit. Proceedings of the ACM on HumanComputer Interaction 4, CSCW1 (2021), 70. https://doi.org/10.1145/3449144

[38] Colin M Gray, Yubo Kou, Bryan Battles, Joseph Hoggatt, and Austin L Toombs. 2018. The Dark (Patterns) Side of UX Design. In Proceedings of the 2018 CHI 
Conference on Human Factors in Computing Systems (Montreal OC, Canada) (CHI '18). ACM, New York, NY, USA, 534:1-534:14. https://doi.org/10.1145/3173574. 3174108

[39] Tony Greening, Judy Kay, and Bob Kummerfeld. 2004. Integrating ethical content into computing curricula. In Proceedings of the Sixth Australasian Conference on Computing Education - Volume 30 (Dunedin, New Zealand) (ACE '04). Australian Computer Society, Inc., Darlinghurst, Australia, Australia, 91-99.

[40] Steve Harrison, Phoebe Sengers, and Deborah Tatar. 2011. Making epistemological trouble: Third-paradigm HCI as successor science. Interacting with computers 23, 5 (Sept. 2011), 385-392. https://doi.org/10.1016/j.intcom.2011.03.005

[41] Justin L Hess and Grant Fore. 2018. A Systematic Literature Review of US Engineering Ethics Interventions. Science and engineering ethics 24, 2 (April 2018), 551-583. https://doi.org/10.1007/s11948-017-9910-6

[42] Jes A Koepfler, Luke Stark, Paul Dourish, Phoebe Sengers, and Katie Shilton 2014. Values \& Design in HCI Education. In CHI '14 Extended Abstracts on Human Factors in Computing Systems (Toronto, Ontario, Canada) (CHI EA '14). ACM, New York, NY, USA, 127-130. https://doi.org/10.1145/2559206.2559231

[43] A C Lorents, J M Maris, J N Morgan, and others. 2006. Ethics of computer use: A survey of student attitudes. Proc. Rom. Acad. Ser. A Math. Phys. Tech. Sci. Inf. Sci. (2006).

[44] Arunesh Mathur, Jonathan Mayer, and Mihir Kshirsagar. 2021. What Makes a Dark Pattern... Dark? Design Attributes, Normative Considerations, and Measurement Methods. (Jan. 2021). https://doi.org/10.1145/3411764.3445610 arXiv:2101.04843 [cs.HC]

[45] Andrew McNamara, Justin Smith, and Emerson Murphy-Hill. 2018. Does ACM's code of ethics change ethical decision making in software development?. In Proceedings of the 2018 26th ACM Joint Meeting on European Software Engineering Conference and Symposium on the Foundations of Software Engineering. ACM, 729-733. https://doi.org/10.1145/3236024.3264833

[46] Keith Miller. 1988. Integrating Computer Ethics into the Computer Science Curriculum. Computer Science Education 1, 1 (Jan. 1988), 37-52. https://doi.org/ 10.1080/0899340880010104

[47] Arvind Narayanan, Arunesh Mathur, Marshini Chetty, and Mihir Kshirsagar 2020. Dark Patterns: Past, Present, and Future. Queueing Systems. Theory and Applications 18, 2 (April 2020), 67-92. https://doi.org/10.1145/3400899.3400901

[48] Don Norman. 2013. The Design of Everyday Things: Revised and Expanded Edition Basic Books.

[49] Michael Quinn Patton. 2014. Qualitative Research \& Evaluation Methods: Integrating Theory and Practice. SAGE Publications.

[50] Jeffrey Saltz, Michael Skirpan, Casey Fiesler, Micha Gorelick, Tom Yeh, Robert Heckman, Neil Dewar, and Nathan Beard. 2019. Integrating Ethics within Machine Learning Courses. ACM Trans. Comput. Educ. 19, 4 (Aug. 2019), 1-26. https: //doi.org/10.1145/3341164

[51] Ben Rydal Shapiro, Amanda Meng, Cody O'Donnell, Charlotte Lou, Edwin Zhao, Bianca Dankwa, and Andrew Hostetler. 2020. Re-Shape: A Method to Teach Data Ethics for Data Science Education. In Proceedings of the 2020 CHI Conference on Human Factors in Computing Systems (Honolulu, HI, USA) (CHI '20). Association for Computing Machinery, New York, NY, USA, 1-13. https://doi.org/10.1145/ 3313831.3376251

[52] Katie Shilton. 2013. Values Levers: Building Ethics into Design. Sci. Technol. Human Values 38, 3 (May 2013), 374-397. https://doi.org/10.1177/0162243912436985

[53] Katie Shilton, Donal Heidenblad, Adam Porter, Susan Winter, and Mary Kendig. 2020. Role-Playing Computer Ethics: Designing and Evaluating the Privacy by Design (PbD) Simulation. Sci. Eng. Ethics (July 2020). https://doi.org/10.1007/ s11948-020-00250-0

[54] Michael Skirpan, Nathan Beard, Srinjita Bhaduri, Casey Fiesler, and Tom Yeh 2018. Ethics Education in Context: A Case Study of Novel Ethics Activities for the CS Classroom. In Proceedings of the 49th ACM Technical Symposium on Computer Science Education (Baltimore, Maryland, USA) (SIGCSE '18). Association for Computing Machinery, New York, NY, USA, 940-945. https://doi.org/10. 1145/3159450.3159573

[55] Michael Skirpan, Jacqueline Cameron, and Tom Yeh. 2018. Ouantified Self: An Interdisciplinary Immersive Theater Project Supporting a Collaborative Learning Environment for CS Ethics. In Proceedings of the 49th ACM Technical Symposium on Computer Science Education (Baltimore, Maryland, USA) (SIGCSE '18). ACM, New York, NY, USA, 946-951. https://doi.org/10.1145/3159450.3159574

[56] Kyle Slager, Ruby Nunez, William Short, and Stacy A Doore. 2021. Computing Ethics Starts on 'Day One': Ethics Narratives in Introductory CS Courses. In Proceedings of the 52nd ACM Technical Symposium on Computer Science Education (Virtual Event, USA) (SIGCSE '21). Association for Computing Machinery, New York, NY, USA, 1282. https://doi.org/10.1145/3408877.3439648

[57] Aimee van Wynsberghe and Scott Robbins. 2014. Ethicist as designer: a pragmatic approach to ethics in the lab. Sci. Eng. Ethics 20, 4 (Dec. 2014), 947-961. https: //doi.org/10.1007/s11948-013-9498-4

[58] Bimlesh Wadhwa, Eng Lieh Ouh, and Benjamin Gan. 2020. How to and how much? Teaching ethics in an interaction design course. (2020).

[59] Marty J Wolf, Don Gotterbarn, and Michael S Kirkpatrick. 2019. ACM Code of Ethics: Looking Back and Forging Ahead. In Proceedings of the 50th ACM Technical
Symposium on Computer Science Education (Minneapolis, MN, USA) (SIGCSE '19). ACM, New York, NY, USA, 801-802. https://doi.org/10.1145/3287324.3287519

[60] Richmond Y Wong, Deirdre K Mulligan, Ellen Van Wyk, James Pierce, and John Chuang. 2017. Eliciting Values Reflections by Engaging Privacy Futures Using Design Workbooks. Proc. ACM Hum. -Comput. Interact. 1, CSCW (Dec. 2017), 111:1-111:26. https://doi.org/10.1145/3134746 